\title{
Examination of the effects of subject anxiety and task difficulty on the outcome of SALT (Suggestive Accelerative Learning and Teaching) techniques
}

\author{
R. F. S. Job and B. Dipamo \\ University of Sydney
}

\begin{abstract}
A noteworthy feature of studies of SALT and suggestopedia is inconsistent results. Both theory and evidence suggest that these techniques may be beneficial to high anxiety learners learning a difficult task but detrimental to low anxiety learners on an easy task. This mix of results may account for many of the inconsistent results in the literature. Thus the role of anxiety is of considerable importance because it may provide an account of damaging inconsistencies and because it may indicate appropriate areas of application. Therefore the present study examined the impact of SALT techniques on the learning of high and low anxiety learners, given both an easy and a difficult task. The results did not support the main hypothesis: overall the SALT techniques produced results which were inferior to conventional learning techniques. Further, SALT techniques were not superior at any level of anxiety or task difficulty. Thus, the present study confirmed the common failure to find SALT techniques superior to conventional learning. Disadvantages of the SALT techniques were identified and suggested that the method may do better with considerably more learner familiarity with the method.
\end{abstract}

The results of a number of reviews (Palmer, 1985; Shaffer, 1979) including the preceding review (Dipamo \& Job, 1990) suggest that SALT (suggestive accelerative learning and teaching) techniques show some promise, but do not live up to Lazanov's (1978) claims. Extreme variation from negative effects to no statistically significant difference to positive effects exists in evaluations.

A number of factors have been implicated in causing this extreme variability of outcomes: study duration, instructor experience and 
commitment, and learner anxiety. From the point of view of practical application, knowledge of the exact role of any of these variables may be of considerable value, because it may allow the determination of conditions under which SALT techniques will be effective. However, study duration, teacher experience and teacher commitment to SALT are confounded. In other words, more committed teachers are more likely to gain more experience (or vice versa) and more likely to commit themselves to longer term studies. Thus, any one of these may account for the apparent influence of the other factors. It is not, at present, clear which of these factors is / are responsible for the observed effects. Furthermore, these effects could arise from the powerful impact of unconscious bias due to teacher expectation (see Rosenthal \& Jacobson, 1968). For this reason the possible moderating variable with the greatest potential for practical application is learner anxiety. The potential for application to the business and consulting sector arises directly from the potential for anxiety to account for the variability of results. For example, variation may arise largely from SALT being effective with high anxiety subjects but harmful to low anxiety subjects. Thus studies which inadvertently used high anxiety subjects would show a positive effect, studies with low anxiety subjects would show negative effects, and studies which used (the typical) mixed population would be unlikely to show a significant effect. If this is correct, the practical implication is that work with generally high anxiety clients should include SALT techniques and work with low anxiety clients should not. This may be readily applicable since certain areas may attract people who tend to be predominantly at one end of the anxiety scale. Alternatively, easily administered tests of anxiety are readily available for screening people for the different procedures.

Thus far these considerations are hypothetical. However, a well established psychological principle - the Yerkes-Dodson Law - predicts that the relaxation and suggestion components (assuming that they reduce anxiety as intended) of SALT or suggestopedia methods would be more beneficial to high or low anxiety learners depending on the difficulty of the task involved.

The Yerkes-Dodson Law identifies a relationship between motivation or anxiety and task difficulty such that higher anxiety will produce better performance on an easy task, whereas lower anxiety (or lower motivation) will result in better performance on a difficult task. A substantial body of evidence supports this law, especially in instances of stressful or anxiety producing motivational states (see Cofer \& Appley, 1964; Hulse, Egeth, \& Deese, 1980). Thus, the relaxation and suggestion components of SALT may be effective for high anxiety subjects in a complex learning task by reducing their anxiety level. However, by this logic SALT would impair the performance of low anxiety subjects in a relatively simple learning situation. 
In addition to this argument based on a law of psychology, evidence directly implicates anxiety as a factor in SALT outcomes. Martin and Schuster (1977; Schuster \& Martin, 1980) studied this issue. They found evidence of trait-state matching: high anxiety subjects performed better when tense and low anxiety subjects performed better when relaxed. This suggests that high anxiety subjects may have become accustomed to their anxiety state, and thus may learn more efficiently in a more anxious state. Similarly, low anxiety subjects may learn better in a low anxiety state.

Relevant theory and evidence along with practical implications indicate that anxiety is an important variable in SALT outcomes. Therefore, an evaluation of SALT techniques was undertaken. It involved a systematic study of high and low anxiety subjects learning easy and difficult tasks.

In order to avoid methodological problems identified in previous studies (see Dipamo \& Job, 1990) control groups were employed with unbiased allocation of subjects and equal time in the experimental situation.

\section{Present Study}

\section{Rational}

The numerous previous studies have produced inconsistent results. It appears likely that unreliable methodology and variations in subject anxiety are two major contributors to the variability of reported results. Consequently the first aim of the present study was to evaluate the relevant techniques while avoiding the methodological flaws identified in the literature.

The second aim was to examine the roles of anxiety and task difficulty. There are two reasons for this aim. First, Schuster \& Martin (1980) have shown that task difficulty and anxiety interact in unusual ways with SALT procedures. However, their study did not resolve the issue since they employed combinations of relaxation and tension prior to and at the time of learning, rather than simply examining relaxation in the SALT procedures. Furthermore, they did not include a group of subjects given no mood manipulation. Thus, the baseline is not known and so the direction of the changes observed is unclear. Second, the Yerkes-Dodson Law (which is well supported by experimental evidence) would suggest that relaxation would help highly anxious subjects on difficult tasks, but impair low anxiety subjects on easy tasks. This may explain some of the observed inter-study variability.

Rather than focus, as most other studies do, on only one or two elements of SALT, such as music and relaxation, this study utilised a number of accelerated learning techniques as outlined in "Superlearning" (Ostrander 
\& Schroeder, 1979). The format used in "Superlearning" was chosen for two reasons. First, it appears to be the most detailed and concise articulation of accelerated learning techniques available; and second, it has been used successfully in the past (Zeiss, 1984; Applegate, 1983 in Schuster \& Gritton, 1986).

\section{Methods}

\section{Subjects}

Subjects were 48 undergraduate students enrolled in a first year psychology course. Credit was given for participation. No attempt was made to control for gender, since previous studies had indicated no gender differences (Schuster \& Mouzon, 1982).

\section{Instruments}

The test instrument consisted of 75 paired associate nouns drawn from a list of 925 nouns (scaled on relevant variables by Paivio et al.(1968)). Easy and difficult tasks were created by selecting words known to be easy or difficult to recall. (The paired associate task involves learning to "associate" two words so that when presented with the first word in a test the subject can reply with the second word).

\section{Procedure}

In order to select appropriate anxiety subjects 83 subjects of both sexes were given the State-Trait Anxiety Inventory (Spielberger, et al., 1968), Form V, under normal classroom conditions. The 24 subjects recording the highest level of state anxiety were selected and randomly assigned to either the control group or experimental group. The same procedure was followed for the 24 subjects recording the lowest level of state anxiety. The state scale was used since Johnson \& Spielberger (1968) had indicated that trait anxiety was impervious to relaxation instructions. In addition, it was hypothesised that since test anxiety is an instance of state anxiety, subjects exhibiting high or low levels of anxiety in the questionnaire would manifest similar levels during the experimental procedure.

Four groups were used, two experimental (one given the difficult, one given the easy task) and two control (difficult and easy). Each group consisted of six high anxiety subjects and six low anxiety subjects, such that each group comprised 12 subjects. (Gender was not specifically controlled, but was randomly assigned. The control groups comprised six males and 18 females, the experimental groups, four males and 20 females.)

Subjects were required to learn 75 paired associate nouns which were presented to them in two lists. A total of 15 minutes were given to learn 
the word associates then subjects were given a 10 minute test in which they were cued with the stimulus word and had to supply the response word. Order of presentation of word pairs in the cued test differed from order of presentation in the learning situation.

All sessions lasted 40 minutes and started with both groups receiving the same instructions as to the nature of the experiment and what was required of them. The control groups then had a six minute, self-relaxation period with soft, melodious music playing in the background. Lights in the room were dimmed and subjects were told to close their eyes and sit back and relax. Following this period, subjects were given a brief, two minute filler task (to equate for time in the situation) after which they were informed they had five minutes to begin learning the list of 75 word pairs in any way they wished. At the end of that time another two minute filler task (to equate for time) followed in which subjects were asked to record the method(s) which they were using to learn the material. Subjects then had a further ten minutes in which to complete the learning task.

The experimental groups were asked to follow a six minute guided, deep muscle relaxation procedure, as outlined by Ostrander \& Schroeder (1979, p.98). This is similar in format to the progressive relaxation methods of Jacobson (1938). The relaxation ended with subjects being given the suggestion that learning was easy, that they could not help but learn using this method. Subjects were then given examples of how mental imagery could be used to connect word pairs. Subjects were also informed of the value of constructing a sentence containing both words and asked to use these techniques to learn the material.

For the experimental group all 75 paired associate words had been recorded on a tape and these were spoken, with oral intonation, at four second intervals. Classical music by Tchaikovsky ("Winter Dreams") played in the background. Subjects had been given two printed lists containing the 75 word pairs, and they read along silently with the recording. This session comprised the "active presentation". In order not to cause confusion instructions on breathing techniques were only given after this session. Once subjects had been given instructions on how to synchronise their breath with the presentation of the music, the "passive review" began. Each word pair was presented over four seconds, followed by a four second pause. During the presentation of the material, subjects were requested to hold their breath and only breathe out and in during the pause. The recorded material then played for another ten minutes, the voice again using oral intonation, during which baroque music (Vivaldi's "Four Seasons") played in the background. In this presentation subjects sat relaxed, with eyes closed and listened to the recording. Instructions had been given to remember the visualisations or sentences made during the active presentation of the material. Individuals are capable of constructing visualisations in four seconds (Bugelski, Kidd \& Segman, 1968). Both 
groups then took a ten minute, cued, immediate recall test. This test was repeated, for all subjects, 7-8 days later to test retention.

\section{Results}

The experimental design included 5 factors: between subjects factors of teaching method (SALT vs control) and anxiety level (high vs low) and three within subject factors of task difficulty (easy/concrete vs difficult/abstract nouns), seven blocks of five words, and time (immediate vs delayed test).

A 5-way analysis of variance (MANOVA in SPSSX) was employed in analysis, reflecting the above design. This analysis revealed a number of expected effects which confirmed the intended manipulations. First, as would be expected more nouns were recalled on the immediate than on the delayed test (means $=42.6$ and 13.9 respectively; $\mathrm{F}=239.4, \mathrm{DF}=1,44$, $\mathrm{p}<.0005)$. Second, as required, the difficult (abstract) nouns were indeed more difficult to recall than the easy (concrete) nouns $(\mathrm{F}=86.1, \mathrm{DF}=1,44$, $\mathrm{p}<.0005)$. However, there was no interaction between anxiety and task difficulty $(\mathrm{F}=.017)$. Nonetheless, anxiety did directly influence performance. High anxiety subjects showed better results than low anxiety subjects $(\mathrm{F}=4.42, \mathrm{DF}=1,44, \mathrm{p}<.041$, see Figure 1$)$.

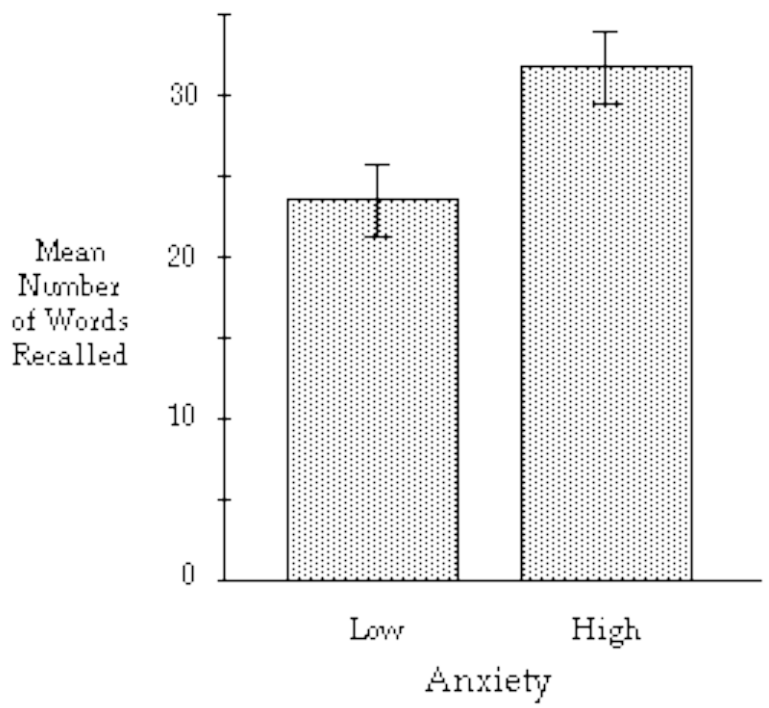

Figure 1: The effect of anxiety on the learning of paired associate nouns (including Standard Error of the Mean) 
Our interest focused on the results relevant to the SALT techniques Overall the SALT techniques proved inferior to conventional teaching methods $(\mathrm{F}=4.57, \mathrm{DF}=1,44, \mathrm{p}<.038$; see Figure 2$)$. Further, the conventional (control) group performed substantially better than the SALT group on the immediate test. However, on the delayed test one week later the difference between the groups had narrowed but still favoured the conventional group. This pattern of results is reflected in a significant SALT versus conventional by immediate versus delayed test interaction $(\mathrm{F}$ $=4.45, \mathrm{DF}=1,44, \mathrm{p}<.041)$. Finally, the SALT method did not cause significantly superior learning in either the high or the low anxiety subjects. Indeed, the means were actually in favour of the conventional group in both cases.

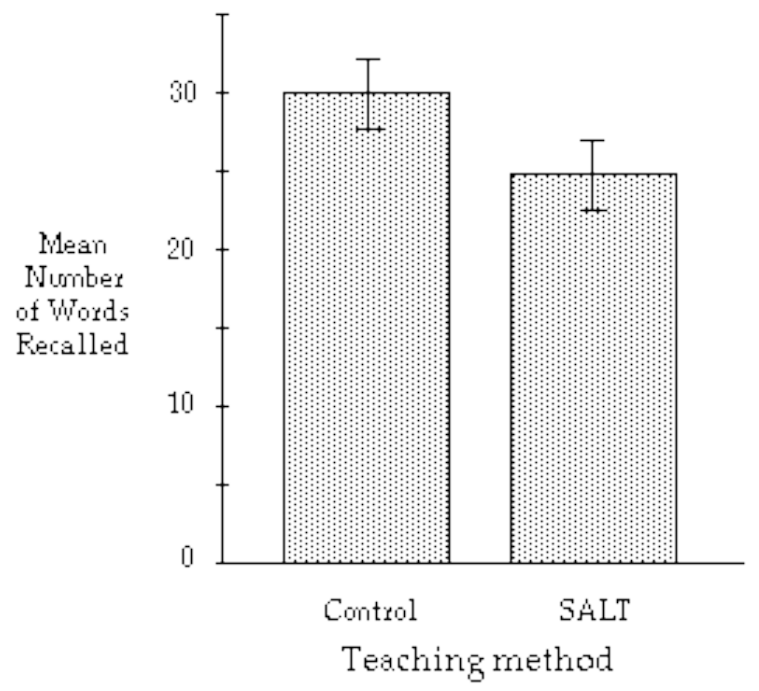

Figure 2: The effect of teaching method on the learning of paired associate nouns (including Standard Error of the Mean).

\section{Concluding Comments}

The present study confirmed the common failure to observe any significant improvement with SALT methods, even when a number of recommended techniques were combined. Furthermore SALT techniques were not differentially effective on either high or low anxiety subjects.

Certain disadvantages of the SALT method were identifiable in the study. These disadvantages may account for the inferior performance of the SALT subjects in the present study. For example, some subjects found the method unfamiliar and distracting. The music itself may also be 
distracting. Experience may help with this, as may the use of music more familiar to the particular learners employed. However, lengthy experience must be used with caution in evaluations because it may have the effect of selecting those subjects who are doing well or like the method, thus creating bias. Finally, the regulated timing has the disadvantage of lack of control which means that subjects cannot skip over material they already know and spend more time on difficult items. Given the potential of certain components of SALT and suggestopedia for improved learning, attempts to eliminate the above problems with the method may prove worthwhile.

\section{References}

Bugelski, B. R., Kidd, E. \& Segman, J. (1968). Image as a mediator in onetrial paired associate learning". Journal of Experimental Psychology, 76, 69-73.

Cofer, C. N. \& Appley, M. H. (1967). Motivation: Theory and Research. 2nd ed. New York: Wiley.

Dipamo, B. \& Job, R. F. S. (1991). A methodological review of studies of SALT (suggestive accelerative learning and teaching) techniques. Australian Journal of Educational Technology, 7(2), 127-143. http: / / www.ascilite.org.au/ajet/ajet7/dipamo.html

Jacobson, E. (1938). Progressive Relaxation. Chicago: University of Chicago Press.

Johnson, D. L. \& Spielberger, C. D. (1968). The effect of relaxation training and the passage of time on measure of state and trait-anxiety. Journal of Clinical Psychology, 24, 20-23.

Lozanov, G. (1978). Suggestology and Outlines of Suggestopedy. NY: Gordon \& Breach.

Martin, D. J. \& Schuster, D. H. (1977). The interaction of trait anxiety and muscle tension in learning. Journal of Suggestive-Accelerative Learning and Teaching, 2 (1\&2), 63-67.

Ostrander, S. \& Schroeder, L. (1979). Superlearning. Melbourne: Souvenir Press (Aust) Pty Ltd.

Paivio, A., Yuille, J. C. \& Madigan, S. A. (1968). Concreteness, imagery and meaningfulness values for 925 nouns. Journal of Experimental Psychology Monograph Supplement, 76(1, Part 2), 1-25.

Palmer, L. L. (1985). SALT with learning disabled and other special need students: a literature review and meta-analysis. Journal of the Society for Accelerated Learning and Teaching, 10(2), 99-129.

Rosenthal, R. \& Jacobson, L. (1968). Pygmalion in the classroom. NY: Holt, Rinehart \& Winston.

Schuster, D. H. \& Gritton, C. E. (1986). Suggestive Accelerative Learning Techniques. NY: Gordon \& Breach. 
Schuster, D. H. \& Martin, D. J. (1980). The effect of biofeedback-induced tension or relaxation, chronic anxiety, vocabulary easiness, suggestion and sex of subject on learning rare English words". Journal of the Society for Accelerated Learning and Teaching, 5(4), 275-287.

Schuster, D. H. \& Mouzon, D. (1982). Music and vocabulary learning. Journal of the Society for Accelerated Learning and Teaching, 7(1), 82-107.

Shaffer, D. (1979). The Lazanov effect: A scientific explanation. Journal of Suggestive-accelerative Learning and Teaching, 4, 177-187.

Spielberger, C. D., Gorsuch, R. L. \& Lushene, R. (1968). Self-Evaluation Questionnaire. Palo Alto, CA: Consulting Psychologist Press.

Zeiss, P. A. (1984). A comparison of the effects of superlearning techniques on the learning of English as a second language. Journal of the Society for Accelerated Learning and Teaching, 9(2), 93-101.

Please cite as: Job, R. F. S. and Dipamo, B. (1991). Examination of the effects of subject anxiety and task difficulty on the outcome of SALT (Suggestive Accelerative Learning and Teaching) techniques. Australian Journal of Educational Technology, 7(2), 144-152.

http: / / www.ascilite.org.au/ajet/ajet7/job.html 